\title{
Patients with treatable malignant diseases - including heart failure - are entitled to specialist care
}

\section{John G.F. Cleland}

ß See related article page 189

$\mathrm{I}$ $\mathrm{n}$ this issue ${ }^{1}$ (see page 189) Ezekowitz and colleagues examine the impact of physician specialty on the outcome of care in patients discharged from hospital with a new diagnosis of congestive heart failure. Their comparison of patients who received no follow-up cardiovascular care, follow-up with a family physician alone or follow-up with a family physician and a specialist in combination suggests that specialist care reduces mortality but not admissions to hospital. Given that the provision of effective and cost-effective care for patients with heart failure presents a difficult challenge for any health care system, this study has important implications. Its methodology, therefore, merits careful consideration.

The researchers used a hospital discharge database to identify 3136 patients who had survived an index admission for new-onset congestive heart failure during a 2-year period in Alberta (1998-2000). Using hospital admissions to select the study cohort seems reasonable, since new cases of congestive heart failure present predominantly in hospital and almost all cases are admitted within a year of diagnosis. ${ }^{2,3}$ However, the number of cases for the Alberta population (2.8 million) suggests an incidence of heart failure of about 1 per 1000 per year, whereas epidemiologic studies suggest an incidence 2-4 times greater., ${ }^{2,4,5}$ This probably reflects the selection of patients with heart failure coded as the primary diagnosis, a methodology that has become conventional but may be flawed. Studies conducted in the United Kingdom suggest it is a toss-up whether heart failure is coded as the primary or the secondary diagnosis. ${ }^{6,7}$ As an example, if a patient is admitted to hospital with myocardial infarction and pulmonary edema and goes on to develop chronic heart failure, some physicians will code the most important continuing problem (heart failure) as the primary diagnosis, whereas others will identify the underlying cause (myocardial infarction) this way. It is likely that patients whose heart failure is coded as a secondary diagnosis have more confounding comorbidities. Excluding such patients makes the study cohort less representative of the clinical population. However, the age and sex distribution of patients included in Ezekowitz and colleagues' sample appear to be epidemiologically representative.
It is unfortunate that the authors confined their interest mainly to cardiovascular follow-up and admissions and paid little attention to the impact of all health care contacts. A third or more of hospital admissions of patients with heart failure are for primarily noncardiovascular problems, ${ }^{8}$ and many patients with heart failure receive cardiovascular care and advice during health care contacts prompted by noncardiovascular complaints. Many patients were reported not to have had any health care visits after discharge, and these patients had the worst outcomes. Presumably, many of these patients died or were readmitted within a few weeks of discharge and did not have an opportunity to seek outpatient follow-up care. Further, it is not clear how patients who did not seek outpatient follow-up until after a second or third admission were handled, but the methodology suggests that these patients would have been included in the group who received cardiovascular care. Although the authors attempted to adjust for some of these problems, their methodology may be biased in favour of (or against) more intensive care.

Patients managed solely by family physicians were less likely to be readmitted to hospital but had poorer survival than those managed jointly by specialists and family physicians. Again, the interpretation of this finding is complex. It is possible that older patients were managed in nursing homes in a more palliative manner and therefore had a lower risk of hospital admission and a higher rate of death. Perhaps sicker patients were more likely to be re-admitted and then referred on to a specialist as suggested by the higher rate of co-morbidities in this group. Moreover, only half of patients with congestive heart failure are likely to have left ventricular systolic dysfunction. ${ }^{9}$ Such patients have poorer survival than those with preserved function but are more likely to receive specialist care. Indeed, men, who are more likely to have left ventricular systolic dysfunction underlying their heart failure, ${ }^{9}$ were more often referred to a specialist in the present study, and women, who are likely not to have left ventricular systolic dysfunction, ${ }^{9}$ had a better prognosis despite being older. If these considerations hold true in Ezekowitz and colleagues' study cohort, their analysis may greatly underestimate the impact of specialist care. 
Randomized controlled trials that compare different models of care generally favour an organized, systematic approach to care, ${ }^{10-13}$ and specialist care in itself may further improve outcomes. ${ }^{14}$ It is logical that community-based integrated care with close specialist involvement should represent the ideal. Heart failure is usually a progressive condition for which surveillance, investigations and treatment are complex. Management needs to move from traditional forms of follow-up toward the type of model used in cancer care, in which planned, periodic, detailed assessment to evaluate progression, regression and recurrence are the norm. If we truly practised evidence-based medicine, patients with heart failure would have symptoms, signs, serum electrolyte levels and renal function assessed at least every 4 months,$^{10}$ as has been done in most successful trials. Less frequent specialist follow-up has never been put to the test. Patients should also have electrocardiograms done periodically to assess heart rhythm (atrial fibrillation is often missed on clinical examination), QRS width (as a guide to need for cardiac resynchronization) and hemoglobin concentrations (given the high prevalence of anemia). Whether assessment for revascularization or implantable defibrillators is in the patient's best interest remains controversial. ${ }^{15,16}$ Changes in cardiac structure and function are a useful guide to prognosis. It is customary to measure disease progression (e.g., tumour size) in other malignant conditions, so why not in heart failure? These follow-up measures can be provided in primary or secondary care, but the person who provides them, whatever their previous training, becomes a specialist.

Clinical trials commonly use hospital admissions as an adverse outcome measure. However, hospital admisssion is, to a large extent, merely a convenient, easily measurable event of only modest relevance to patients with heart failure. ${ }^{17}$ Although hospital admission is used as a surrogate measure for survival, symptom control and costs of treatment, these can measured more accurately by other means. For example, asking patients about the severity of their symptoms on an absolute scale (to avoid the problems inherent in asking patients about changes in symptoms) and measuring the number of days in hospital would yield findings that are more accurate and more relevant to patients. Indeed, hospital admission is not intrinsically a bad thing for most patients. Patients with severe acute symptoms expect to be admitted. Patients with chronic severe symptoms are usually happy to be admitted for investigation or adjustment of treatment for short periods if they believe it has a chance of improving how they feel in the long term and reducing the risk of a future crisis. Ezekowitz and colleagues' study is not the first to suggest that improved medical care may reduce mortality but possibly increase rates of hospital admission..$^{18}$ Clinical trials should be selective about which admissions are regarded as adverse, or else cease to use hospital admissions as an outcome altogether (although perhaps not the number of days in hospital). Likewise, clinical services should not use solely the number or the duration of hospital admissions as a marker of adverse outcomes. Increasing the threshold for admission and premature discharge are not in patients' best interests. Hospital bed-days may be a better marker, since this reflects both the number and the duration of admissions. Timely admission and good discharge planning are rewarded by this outcome measure, but not by measuring either component alone.

Ezekowitz and colleagues suggest that randomized controlled trials comparing a strategy of integrated care with the existing haphazard structures are impossible. This is not the case. Well-designed clinical trials can be done, and they will help ensure that optimal care is delivered, with the elimination of harmful and redundant practices and the adoption of effective, affordable care. What this study and others suggest is that expert-led, integrated care is the "gold standard" against which other strategies should be tested. We already know that haphazard care fails many patients and is expensive, but improvements are inhibited by the fear that specialists will throw all the available technology at patients regardless of proof of safety, efficacy or affordability. Aspirin has not been shown to be safe, ${ }^{19}$ revascularization has not been shown to be effective ${ }^{15}$ and implantable defibrillators have not been shown to be affordable in patients with heart failure. ${ }^{16}$ Specialists must show that they can act responsibly as guardians of health care resources, which must be used wisely to gain the trust of those who fund health care.

Dr. Cleland is Professor of Cardiology, University of Hull, Kingston-upon-Hull, UK. Competing interests: None declared.

\section{References}

1. Ezekowitz JA, van Walraven C, McAlister FA, Armstrong PA, Kaul P. Impact of specialist follow-up in outpatients with congestive heart failure. CMAJ 2005;172(2):189-94.

2. Cleland JG, Khand A, Clark AC. The heart failure epidemic: Exactly how big is it? Eur Heart 7 2001; 22(8):623-6.

3. Johansson S, Wallander M-W, Ruigomez A, Rodriguez LAC. Incidence of newly diagnosed heart failure in UK general practice. Eur 7 Heart Fail 2001; 2:225-31.

4. Roger VI, Weston SA, Redfield MM, Hellermann-Homan JP, Killian J, Yawn BP, et al. Trends in heart failure incidence and survival in a community-based population. 7AMA 2004;292(3):344-50.

5. Murphy NF, Simpson CR, McAlister FA, Stewart S, MacIntyre K, Kirkpatrick $M$, et al. National survey of the prevalence, incidence, primary care burden, and treatment of heart failure in Scotland. Heart 2004;90(10):1129-36.

6. Brown A, Cleland JG. Influence of concomitant disease on patterns of hospitalisation in patients with heart failure discharged from Scottish hospitals in 1995. Eur Heart 7 1998;19(7):1063-9.

7. Cleland JG, Gemmel I, Khand A, Boddy A. Is the prognosis of heart failure improving? Eur 7 Heart Fail 1999;1:229-41.

8. Khand AU, Gemmell I, Rankin AC, Cleland JG. Clinical events leading to the progression of heart failure: Insights from a national database of hospital discharges. Eur Heart 7 2001; 22:153-64.

9. Cleland JG, Swedberg K, Follath, et al, for the Study Group on Diagnosis of the Working Group on Heart Failure of the European Society of Cardiology, Freemantle N, Eastaugh J, Mason J. The EuroHeart Failure Survey Programme: Survey on the Quality of Care Among Patients with Heart Failure in Europe. Part 1: Patient Characteristics and Diagnosis. Eur Heart 7 2003; 24:422-63.

10. Cleland JG, Lalukota K. How often should a patient with heart failure be seen by a specialist. A review of the evidence? Eur 7 Heart Fail [In press]

11. McAlister FA, Stewart S, Ferrua S, McMurray JJV. Multidisciplinary strategies for the management of heart failure patients at high risk for readmission. f Am Coll Cardiol 2004;44:810-9. 
12. Gustafsson F, Arnold JMO. Heart failure clinics and outpatient management: review of the evidence and call for quality assurance. Eur Heart 7 2004;25 (1596): 1604 .

13. Louis AA, Turner T, Gretton M, Baksh A, Cleland JG. A systematic review of telemonitoring for the management of heart failure. Eur 7 Heart Fail 2003 ; 5:583-90.

14. Fonarow GC. Heart failure disease management programs: not a class effect. Circulation 2004;110(23):3506-8.

15. Cleland JG, Freemantle N, Ball SG, Bonser RS, Camici PG, Chattopadhyay $\mathrm{S}$, et al. The heart failure revascularization trial (HEART): rationale design and methodology. Eur 7 Heart Fail 2003;5(3):295-303.

16. Shelton RJ, Velavan P, Nikitin NP, Coletta AP, Clark AL, Rigby AS, Freemantle N, Cleland JGF. Clinical trials update from the American Heart Association meeting: ACORN-CSD, primary care trial of chronic disease management, PEACE, CREATE, SHIELD, A-HeFT, GEMINI, vitamin E meta-analysis, ESCAPE, CARP, and SCD-HeFT cost effectiveness study. Eur 7 Heart Fail. In press.
17. Cleland JG. How to assess new treatments for the management of heart failure: composite scoring systems to assess the patients' clinical journey. Eur 7 Heart Fail 2002; 4(3):243-7.

18. Louis AA, Balk AHMM, Janssens U, Cleland JGF. Telemonitoring for patients with heart failure at high risk for readmission and death. TheTrans-European Network Home Monitoring Study (TEN-HMS). Eur 7 Heart Failure 2003;145(suppl) [abstract].

19. Cleland JG. Is aspirin "The Weakest Link" in cardiovascular prophylaxis? The surprising lack of evidence supporting the use of aspirin for cardiovascular disease. Prog Cardiovasc Dis 2002;44:275-92.

Correspondence to: Dr. John Cleland, European Journal of Heart Failure, Academic Cardiology, University of Hull, Castle Hill Hospital, Kingston-upon-Hull, UK, HU16 5JQ;

fax 441482 624085; j.g.Cleland@hull.ac.uk

\section{Clinical PRACTICE Guidelines}

FOR THE CARE AND TREATMENT OF BREAST CANCER

In February 1998 CMAJ and Health Canada published 10 clinical practice guidelines for the care and treatment of breast cancer, along with a lay version designed to help patients understand more about this disease and the recommended treatments. These guidelines are currently being revised and updated, and the series is being extended to cover new topics. The complete text of the new and updated guidelines is available at eCMA):

\section{www.cmaj.ca/cgi/content/full/158/3/DC1}

REVISED:

Guideline 3: Mastectomy or lumpectomy? The choice of operation for clinical stages I and II breast cancer [July 23, 2002]

Guideline 5: The management of ductal carcinoma in situ [Oct. 2, 2001]

Guideline 6: Breast radiotherapy after breastconserving surgery [Feb. 18, 2003]

Guideline 7: Adjuvant systemic therapy for women with node-negative breast cancer [Jan. 23, 2001]

Guideline 8: Adjuvant systemic therapy for women with node-positive breast cancer [Mar. 6, 2001]

Guideline 10: The management of chronic pain in patients with breast cancer [Oct. 30, 2001]
NEW:

Guideline 11: Lymphedema [Jan. 23, 2001]

Guideline 12: Chemoprevention [June 12, 2001]

Guideline 13: Sentinel node biopsy [July 24, 2001]

Guideline 14: The role of hormone replacement therapy in women with a previous diagnosis of breast cancer [Apr. 16, 2002]

Guideline 15: Treatment for women with stage III or locally advanced breast cancer [Mar. 16, 2004]

Guideline 16: Locoregional post-mastectomy radiotherapy [Apr. 13, 2004] 